\title{
Consensus guidelines for perioperative point-of-care ultrasound: the devil is in the (implementation) details
}

\author{
Scott J. Millington, MD FRCPC (B)
}

Received: 1 September 2020/Accepted: 2 September 2020/Published online: 20 November 2020

(C) Canadian Anesthesiologists' Society 2020

In this issue of the Journal, Meineri et al. add to the global point-of-care ultrasound (POCUS) momentum by presenting Canadian training and performance guidelines for perioperative ultrasound. ${ }^{1}$ Their sensible consensus document, very much in-line with similar guidelines in other countries ${ }^{2}$ and for other specialties, ${ }^{3}$ is highlighted by recommendations for:

- What to learn: basic cardiac, lung, airway, gastric, and abdominal ultrasound

- How to learn: live and off-line education, including a suggestion for 100 practice exams

- How to teach: dedicated supervision and competency assessment for learners

- How to be safe: archiving of ultrasound images, reporting of relevant findings, and a quality assurance system.

Point-of-care ultrasound (or resuscitative ultrasound, or critical care ultrasound, or whichever term you prefer) has become an essential element in caring for acutely unwell patients in anesthesia, but also in critical care, emergency medicine, internal medicine, and trauma care. Each of these disciplines list POCUS objectives amidst their core competencies or training experiences for Royal College certification, ${ }^{\mathrm{A}, \mathrm{B}, \mathrm{C}, \mathrm{D}, \mathrm{E}}$ yet the current landscape of ultrasound education in Canada remains highly variable. ${ }^{4}$

Many, if not most, training programs across all acute care specialties do not live up to even the basic safety requirements as outlined in this consensus document, lacking fundamental elements as basic as the ability to

S. J. Millington, MD FRCPC ( $₫)$

Intensive Care, The University of Ottawa/The Ottawa Hospital,

Ottawa, ON, Canada

e-mail: smillington@toh.ca record and to permanently archive an ultrasound image or the availability of a content expert to review it with. This situation would be considered intolerable in any other circumstance: imagine a critical care program without an organized approach to teaching bronchoscopy, or an emergency medicine program without a well-delineated pathway to learning airway management.

This practical consensus document should serve as a call to arms for the professionalization of POCUS training in all acute care specialties. Hospitals should prioritize and fund a point-of-care image archiving solution for all relevant departments. Such systems are readily available and relatively inexpensive, and image archiving is the foundational element which permits POCUS to be taught and performed safely and efficiently. The Royal College should continue to encourage POCUS learning in the era of competence by design by creating entrustable professional activities (EPAs); luckily, ultrasound skills fit well into the EPA mould as discrete and repeatable tasks. Department heads should devote human resources to solving this problem, as there is no substitute for having physicians on staff with POCUS skills and the protected time to teach it while providing quality assurance. Program directors should elevate the ultrasound skillset to the same level as other core competencies for their training programs, dedicating adequate academic time for instruction and practice.

Numerous problems exist. What to do about staff physicians who don't feel comfortable with POCUS? How to handle incoming waves of medical students, arriving from institutions that teach ultrasound skills with variable degrees of vigour (and rigour)? Which learners will be allowed to scan independently, and when? There are no easy answers here, but clearly these issues are better approached from a position of relative strength, equipped 
with a residency training program that teaches POCUS properly.

Most of all, we should all adhere to published Canadian POCUS guidelines - after all, they currently exist for critical care, ${ }^{3}$ for emergency medicine, ${ }^{5}$ for internal medicine, ${ }^{6}$ and now for anesthesia. ${ }^{1}$

\section{Footnotes}

A. Royal College of Physicians and Surgeons of Canada. Anesthesiology Competencies. Available from URL: http://www.royalcollege.ca/rcsite/documents/ibd/anes thesiology-competencies-e.pdf (accessed September 2020).

B. Royal College of Physicians and Surgeons of Canada. Critical Care Medicine Competencies. Available from URL http://www.royalcollege.ca/rcsite/documents/ibd/ critical-care-medicine-competencies-e.pdf (accessed September 2020).

C. Royal College of Physicians and Surgeons of Canada. Emergency Medicine Competencies. Available from URL: http://www.royalcollege.ca/rcsite/documents/ $\mathrm{ibd} /$ emergency-medecine-competencies-e.pdf

D. Royal College of Physicians and Surgeons of Canada. Internal Medicine Training Experiences. Available from URL: http://www.royalcollege.ca/rcsite/documents/ibd/ internal-medecine-rte-training-experiences-e.pdf (accessed September 2020).

E. Royal College of Physicians and Surgeons of Canada. Competency Training Requirements for the Area of Focused Competence in Trauma General Surgery. Available from URL: http://www.royalcollege.ca/ rcsite/documents/ibd/trauma-general-surgery-ctr-e.pdf (accessed September 2020).

\section{Lignes directrices consensuelles pour l'échographie périopératoire ciblée : le diable est dans les détails (de la mise en ouvre)}

Dans ce numéro du Journal, Meineri et coll. se joignent à l'élan mondial pour l'échographie ciblée (ou au point d'intervention, POCUS) en présentant des lignes directrices canadiennes pour la formation en échographie périopératoire et son application. ${ }^{1}$ Leur document consensuel pratique, conforme aux lignes directrices similaires existant dans d'autres pays ${ }^{2}$ et pour d'autres spécialités, ${ }^{3}$ souligne des recommandations concernant:
- Ce qu'il faut apprendre : l'échographie cardiaque, pulmonaire, respiratoire, gastrique et abdominale de base

- Comment l'apprendre : la formation en direct et 'hors ligne', y compris 100 examens pour pratiquer suggérés

- Comment l'enseigner : supervision et évaluation des compétences dédiées pour les étudiants

- Comment assurer la sécurité : archivage d'images échographiques, communication des résultats pertinents et système d'assurance de la qualité.

L'échographie ciblée (ou échographie de réanimation, échographie de soins intensifs, peu importe votre terme de prédilection) est devenue un élément essentiel des soins aux patients gravement malades en anesthésie, mais aussi aux soins intensifs, en médecine d'urgence, en médecine interne et en soins de traumatologie. Chacune de ces disciplines énumère les objectifs de l'échographie ciblée parmi ses compétences clés ou expériences de formation pour la certification du Collège royal. ${ }^{\text {A,B,C,D,E }}$ Pourtant, le paysage actuel de l'enseignement de l'échographie au Canada demeure très variable. ${ }^{4}$

Bon nombre, sinon la plupart, des programmes de formation dans toutes les spécialités de soins aigus ne sont pas à la hauteur des exigences de sécurité les plus fondamentales telles qu'énoncées dans ce document consensuel. En effet, les programmes actuels ne disposent pas d'éléments fondamentaux aussi élémentaires que la capacité d'enregistrer et d'archiver de façon permanente une image échographique, ou la disponibilité d'un expert pour examiner les images. Cette situation serait inacceptable en toute autre circonstance : imaginez un programme de soins intensifs ne possédant pas d'approche organisée pour enseigner la bronchoscopie, ou un programme de médecine d'urgence ne proposant pas de parcours de formation bien défini pour apprendre la prise en charge des voies aériennes.

Ce document de consensus pratique devrait servir de cri de ralliement pour la professionnalisation de la formation en échographie ciblée dans toutes les spécialités de soins aigus. Les hôpitaux devraient prioriser et financer un système d'archivage des images d'échographie disponible à tous les services concernés. De tels systèmes sont facilement disponibles et relativement peu coûteux, et l'archivage d'images est l'élément fondamental qui permet à l'échographie ciblée d'être enseignée et réalisée de façon sécuritaire et efficace. À l'ère de la compétence par la conception, le Collège royal devrait continuer à encourager l'apprentissage de l'échographie ciblée en créant des actes professionnels non supervisés (APNS); heureusement, les compétences en échographie s'inscrivent bien dans le cadre des APNS étant donné leur caractère à la fois discret et reproductible. Les chefs de département devraient dédier 
des ressources humaines à la résolution de ce problème, car nous ne pouvons nous passer de médecins possédant des compétences en échographie ciblée et de leur temps protégé pour l'enseigner tout en fournissant une évaluation de la qualité. Les directeurs de programme devraient considérer les compétences en échographie au même niveau que les autres compétences de base de leurs programmes de formation, en consacrant suffisamment de temps universitaire à leur enseignement et à leur pratique.

De nombreux problèmes persistent. Que faire des médecins en pratique qui ne sont pas à l'aise avec l'échographie ciblée? Comment gérer les vagues d'étudiants en médecine provenant d'institutions diverses qui enseignent les compétences en échographie avec un enthousiasme (et une rigueur) inégal? Quels étudiants autoriserons-nous à faire une échographie seuls, et quand? Il n'y a pas de réponse facile à cette question, mais il est clair qu'il sera plus facile d'y répondre d'une position de force relative, équipés d'un programme de résidence qui enseigne adéquatement l'échographie ciblée.

Par-dessus tout, nous devrions tous adhérer aux lignes directrices canadiennes publiées sur l'échographie ciblée après tout, à l'heure actuelle elles existent pour les soins intensifs, ${ }^{3}$ pour la médecine d'urgence, ${ }^{5}$ pour la médecine interne, ${ }^{6}$ et maintenant pour l'anesthésie. ${ }^{1}$

Disclosures None.

Funding statement None.

Editorial responsibility This submission was handled by Dr. Philip M. Jones, Associate Editor, Canadian Journal of Anesthesia.
Déclaration de financement: Aucune.

Editorial responsibility Cet article a été traité par Dr Philip M. Jones, rédacteur adjoint, Journal canadien d'anesthésie.

\section{References}

1. Meineri $M$, Arellano $R$, Bryson $G$, et al. Canadian recommendations for training and performance in basic perioperative point-of-care ultrasound: recommendations from a consensus of Canadian anesthesiology academic centres. Can J Anesth 2021; DOI: https://doi.org/10.1007/s12630-020-01867-2.

2. The Association of Anaesthetists of Great Britain \& Ireland; The Royal College of Anaesthetists; The Intensive Care Society. Ultrasound in anaesthesia and intensive care: a guide to training - July 2011. Available from URL: https://anaesthetists.org/Home/ Resources-publications/Guidelines/Ultrasound-in-anaesthesia-andintensive-care-a-guide-to-training (accessed September 2020).

3. Arntfield $R$, Millington $S$ Ainsworth $C$, et al. Canadian recommendations for critical care ultrasound training and competency. Can Respir J 2014; 21: 341-5.

4. Mok D, Schwarz SK, Rondi K. Point-of-care ultrasonography in Canadian anesthesiology residency programs: a national survey of program directors. Can J Anesth 2017; 64: 1023-36.

5. Olszynski P, Kim D, Chenkin J, Rang L. The core emergency ultrasound curriculum project: a report from the Curriculum Working Group of the CAEP Emergency Ultrasound Committee. CJEM 2018; 20: 176-82.

6. Ma IW, Arishenkoff $S$, Wiseman J, et al. Internal medicine pointof-care ultrasound curriculum: consensus recommendations from the Canadian Internal Medicine Ultrasound (CIMUS) Group. J Gen Intern Med 2017; 32: 1052-7.

Publisher's Note Springer Nature remains neutral with regard to jurisdictional claims in published maps and institutional affiliations.

Déclaration: Aucune. 